\title{
Targeted Folate Receptor $\beta$ Fluorescence Imaging as a Measure of Inflammation to Estimate Vulnerability Within Human Atherosclerotic Carotid Plaque
}

\author{
Nynke A. Jager ${ }^{1,2}$, Johanna Westra², Gooitzen M. van Dam ${ }^{1}$, Nato Teteloshvili², René A. Tio ${ }^{3}$, Jan-Cees Breek ${ }^{4}$, \\ Riemer H.J.A. Slart ${ }^{5}$, Hendrikus Boersma ${ }^{6}$, Phillip S. Low ${ }^{7}$, Marc Bij1 $1^{2}$, and Clark J. Zeebregts ${ }^{1}$ \\ ${ }^{1}$ Department of Surgery, University Medical Center Groningen, University of Groningen, Groningen, The Netherlands; ${ }^{2}$ Department of \\ Rheumatology and Clinical Immunology, University Medical Center Groningen, University of Groningen, Groningen, The Netherlands; \\ ${ }^{3}$ Department of Cardiology, University Medical Center Groningen, University of Groningen, Groningen, The Netherlands; ${ }^{4}$ Department of \\ Surgery, Martini Hospital, Groningen, The Netherlands; ${ }^{5}$ Department of Nuclear Medicine and Molecular Imaging, University Medical \\ Center Groningen, University of Groningen, Groningen, The Netherlands; ${ }^{6}$ Department of Clinical Pharmacy, University Medical Center \\ Groningen, University of Groningen, Groningen, The Netherlands; and ${ }^{7}$ Department of Chemistry, Purdue University, Purdue, Indiana
}

The probability of atherosclerotic plaque rupture and its thrombotic sequelae are thought to be increased at sites of macrophage accumulation. Folate receptor $\beta(\mathrm{FR}-\beta)$ is present on activated macrophages but not on quiescent macrophages or other immune cells. By conjugating the ligand folate with a fluorescent contrast agent, fluorescein isothiocyanate (FITC), we aimed to explore the potential role of FR- $\beta$ fluorescence imaging in the distinction of vulnerable sites from more stable regions. Methods: Carotid specimens were taken from 20 patients and incubated with folate-FITC for $30 \mathrm{~min}$. Ex vivo fluorescence imaging was performed to determine the exact location of folateFITC uptake. Sections displaying regions of high uptake (determined as hot spots) were compared with sections showing low uptake (cold spots) through immunohistochemistry and real-time quantitative reverse-transcription polymerase chain reaction for FR- $\beta$. Results: Hot spots showed significantly higher folate-FITC uptake than cold spots $(P<0.001)$. Hot spots tended to contain more macrophages and areas of hypoxia than cold spots. A positive correlation between messenger RNA levels of CD68 (marker for macrophages), FR- $\beta$ ( $r=0.53, P=0.045)$, and hypoxia-inducible factor-1 $\alpha$ expression (marker for intraplaque hypoxia; $r=0.55, P=0.034$ ) was found. Conclusion: Compared with areas with low folate-FITC uptake, areas of high folate-FITC uptake within human atherosclerotic plaques had an increased number of activated macrophages and higher areas of hypoxia. These characteristics of vulnerability imply that molecular imaging of FR- $\beta$ through folate conjugates might be a good indicator for plaque vulnerability in future noninvasive imaging studies.

Key Words: carotid artery; atherosclerotic plaque; vulnerability; folate receptor- $\beta$; fluorescence imaging

J Nucl Med 2012; 53:1222-1229

DOI: 10.2967/jnumed.111.099671

Received Oct. 20, 2011; revision accepted Mar. 12, 2012.

For correspondence or reprints contact: Clark J. Zeebregts, Division of Vascular Surgery, Department of Surgery, University Medical Center Groningen, PB 30.001, 9700 RB Groningen, The Netherlands.

E-mail: czeebregts@hotmail.com

Published online Jun. 29, 2012.

COPYRIGHT (C) 2012 by the Society of Nuclear Medicine and Molecular Imaging, Inc.
A therosclerosis can be regarded as a progressive inflammatory process of the arterial wall ultimately leading to the formation of plaques. Currently, cardiovascular atherosclerotic disease is the leading cause of death in men and women in the western world (1). Atherosclerotic thickening of the vascular wall may compromise end-organ blood flow, but the greatest risk for clinical events is present in patients with an unstable plaque. Rupture of such a vulnerable plaque is one of the predominant underlying processes in the pathogenesis of both myocardial infarction and ischemic stroke. Histologic assessment of ruptured plaques has shown several distinctive features, including inflammatory cell infiltration and increased adventitial and plaque neovascularization (2). Currently, indications for interventional treatment are based on clinical symptomatology and degree of stenosis $(>70 \%)$ $(3,4)$. However, it has been recognized that plaque vulnerability rather than plaque size or severity of stenosis is important in assessments of the risk that plaque will rupture (5). In this context, the extent and location of plaque inflammation appear to be key factors in determining plaque vulnerability (6). In current clinical practice, anatomic imaging modalities, such as B-mode duplex ultrasound, CT angiography scanning, and MRI, can identify stenotic plaques of the internal carotid artery, but they give little or no information regarding molecular and cellular processes $(5,6)$.

Previous studies have shown that inflammation is related to the accumulation of activated macrophages at sites of plaque rupture (7). For this reason, the activated macrophage has emerged as an important diagnostic and therapeutic target for atherosclerosis $(8,9)$. The folate receptor $\beta(\mathrm{FR}-\beta)$ is present on activated macrophages but not on quiescent macrophages or other immune cells (10-12). The folate receptor is a 38-kDa glycosylphosphatidylinositol-anchored protein that binds to the vitamin folic acid with high affinity $(<1 \mathrm{nM})(12)$.

By visualizing the FR- $\beta$ with an optical fluorescent contrast agent, activated macrophages can be detected by 
optical imaging. As such, van Dam et al. conjugated fluorescein isothiocyanate (FITC) with folate (i.e., folateFITC) to identify tumor processes, because several solid tumors express folate receptor $\alpha(\mathrm{FR}-\alpha)$ (13). Similarly, Ayala-López et al. showed that a folate ${ }^{99 \mathrm{~m} T c-E C 20}$ compound binds to the FR- $\beta$ and therefore localizes folate in apolipoprotein E knock-out mice fed a Western diet (11).

Vulnerable plaques are also characterized by intraplaque hypoxia (14). The transcription factor hypoxia-inducible factor- $1 \alpha(\mathrm{HIF}-1 \alpha)$ is regarded as the major oxygen homeostasis regulator. HIF- $1 \alpha$ plays an important role in inflammatory reactions and in angiogenesis and is involved in tumor growth leading to resistance to radiotherapy and chemotherapy $(15,16)$.

The aim of this pilot study was to explore the potential of fluorescence-labeled folate as a macrophage activity marker in the vessel wall. Thus, we investigated whether areas of high folate-FITC uptake reflect the presence of an increased number of activated macrophages, characteristic of an unstable plaque in the internal carotid artery. In addition, we investigated HIF- $1 \alpha$ expression in atherosclerotic plaques and compared this to the distribution of FR- $\beta$ within the plaque to confirm the relationship between hypoxia and plaque vulnerability. These data will contribute to the development of optical imaging of the carotid artery in the near future that could be used as a noninvasive imaging method to detect and quantify the vulnerability of atherosclerotic plaques.

\section{MATERIALS AND METHODS}

\section{Study Design}

Between October 2008 and April 2009, carotid specimens were taken from 20 patients. These patients underwent open carotid surgery in 2 hospitals (University Medical Center Groningen [UMCG] and Martini Hospital, Groningen, The Netherlands). From these patients, atherosclerotic plaques were obtained by means of a carotid endarterectomy of the internal carotid artery using standard techniques. Patients who underwent surgical treatment were symptomatic (i.e., with a history of cerebrovascular accident, transient ischemic attack, or amaurosis fugax) who had a stenosis of $70 \%-99 \%$ as detected by duplex ultrasound. Also, asymptomatic patients with a stenosis of $80 \%-99 \%$ were eligible for surgical treatment (UMCG only). Risk factors such as smoking status, obesity (body mass index), hyperlipidemia, hypertension, and diabetes mellitus were recorded. Hypertension was defined as systolic arterial pressure above $140 \mathrm{~mm} \mathrm{Hg}$ or diastolic arterial pressure above $90 \mathrm{~mm} \mathrm{Hg}$ or the use of antihypertensive drugs, prescribed with the aim to reduce blood pressure (17). Hyperlipidemia was diagnosed if plasma total cholesterol exceeded $6.21 \mathrm{mmol} / \mathrm{L}$, plasma low-density lipoprotein cholesterol exceeded $3.36 \mathrm{mmol} / \mathrm{L}$, plasma triglycerides exceeded $2.26 \mathrm{mmol} / \mathrm{L}$, or if the patient used lipid-lowering drugs (17). The study was approved by the Institutional Review Board of each hospital, and informed consent was obtained from all patients.

\section{Carotid Endarterectomy Sample Collection and Time Path of Study}

The carotid samples were collected during carotid endarterectomy. The samples were immediately transported in a phosphate buffer on ice to the IVIS Spectrum (Caliper Life Sciences), with a transport time of $21 \pm 5 \mathrm{~min}$ at the UMCG hospital and $50 \pm$ $14 \mathrm{~min}$ at the Martini hospital. After the samples were collected and transported, imaging with the IVIS Spectrum was performed after determining the optimal incubation time and concentration (Supplemental Fig. 1; supplemental materials are available online only at http://jnm.snmjournals.org). To validate the folate-FITC signal, protein levels (immunohistochemistry, immunofluorescence) and gene levels (reverse-transcription polymerase chain reaction [RT-PCR]) were measured.

\section{Imaging of Plaque}

Ex vivo fluorescence imaging was performed to determine the exact location at which the folate-FITC (molecular weight, 916 $\mathrm{kDa}$, Supplemental Fig. 2) uptake had taken place within the plaque. Before imaging, the concentration and incubation time were determined by making a serial dilution. Several concentrations were tested. Because the highest intensity was found at an incubation time of $30 \mathrm{~min}$ in $2 \mathrm{~mL}$ of folate-FITC in phosphate-buffered saline and a folate-FITC:phosphate-buffered saline dilution of 1:10 (10 $\mu \mathrm{g} / \mathrm{mL}$ ), we considered these the ideal concentration and incubation time. Fluorescence images were obtained with a commercial imaging system with an ultra-sensitive charge-coupled device camera mounted on a light-tight black chamber (IVIS Spectrum). In this imaging system, we used wavelengths of $500 \mathrm{~nm}$ (excitation wave) and $540 \mathrm{~nm}$ (emission wave) for every sample. To suppress autofluorescence from surrounding tissue, spectral unmixing algorithms were used. The technique of fluorescence imaging with the system has been described previously (18). Sections were taken from locations with the following folate-FITC uptake: high intensity ( $>5.00 \mathrm{e}^{-4}$ radiance, photons $/ \mathrm{s} / \mathrm{cm}^{2} / \mathrm{sr}$; so-called hot spots), medium intensity $\left(2.00 \mathrm{e}^{-4}\right.$ to $5.00 \mathrm{e}^{-4}$ radiance, photons $/ \mathrm{s} / \mathrm{cm}^{2} / \mathrm{sr}$; medium spots $)$, and low intensity $\left(<2.00 \mathrm{e}^{-4}\right.$ radiance, photons $/ \mathrm{s} / \mathrm{cm}^{2} / \mathrm{sr}$; cold spots). Cold spots served as negative control tissue. Images acquired were processed using Living Image software (version 3.0; Caliper Life Sciences).

\section{Validation of Folate-FITC Labeling}

To investigate whether FR- $\beta$ is present on activated macrophages and whether folate-FITC binds to FR- $\beta$, we repeated the experiment as described by Xia et al. (10). Macrophages expressed in synovial fluid of rheumatoid arthritis patients are considered to be activated because proinflammatory or regulatory cytokines and growth factors, tumor necrosis factor- $\alpha$, granulocyte-macrophage colony-stimulating factor, chemokines and chemoattractants, metalloproteinases, and neopterin are all overexpressed (19). Synovial fluid was obtained from patients with rheumatoid arthritis, who had given informed consent, and mononuclear fraction was collected by Ficoll gradient separation. Cells were stained with anti-CD11bPE (BD Biosciences) to distinguish macrophages from other immune cells. To demonstrate the presence of FR- $\beta$ on the macrophages, the receptor was visualized using biotinylated antihuman FR- $\beta$ antibody (R\&D Systems). Streptavidine-allophycocyanine (APC) (Biolegend) was used as secondary antibody. In addition, these cells were incubated with folate-FITC ( $100 \mathrm{nM}, 0.05 \mu \mathrm{g} / \mathrm{mL}$ ), and to test if this was specific binding, we also incubated with a 100-fold excess of free folic acid (no. 1.03984; Merck).

\section{Immunohistochemistry}

For the first 15 patients, biopsy samples were taken through the plaque at the sites of hot, medium, and cold spots represented on the IVIS Spectrum image. Subsequently, a new fluorescence 
TABLE 1

Baseline Characteristics and Risk Factors for Atherosclerosis

\begin{tabular}{lc}
\hline \multicolumn{1}{c}{ Characteristic } & Patients $(n=20)$ \\
\hline Men & $17(85)$ \\
\hline Age $(\mathrm{y})$ & $69 \pm 9$ \\
Body mass index $\left(\mathrm{kg} / \mathrm{m}^{2}\right)$ & $25 \pm 5$ \\
Smoking status & $8(40)$ \\
$>1$ pack a day & $3(38)$ \\
$\leq 1$ pack a day & $5(62)$ \\
Hypertension & $18(90)$ \\
Systolic blood pressure $(\mathrm{mm} \mathrm{Hg})$ & $152 \pm 21$ \\
Diastolic blood pressure $(\mathrm{mm} \mathrm{Hg})$ & $83 \pm 11$ \\
\hline Use of antihypertensives & $18(90)$ \\
\hline Hyperlipidemia & $12(60)$ \\
Use of lipid-lowering drugs & $19(95)$ \\
Low-density lipoprotein $(\mathrm{mmol} / \mathrm{L})$ & $3.2 \pm 1.1$ \\
High-density lipoprotein $(\mathrm{mmol} / \mathrm{L})$ & $1.1 \pm 0.4$ \\
Triglycerides (mmol/L) & $1.5 \pm 0.7$ \\
Diabetes mellitus & $1(5)$ \\
& \\
\hline Data are mean \pm SD or $n$, with percentages in parentheses.
\end{tabular}

image was obtained to ensure that the region was hot, medium, or cold. Next, the biopsy samples were embedded in paraffin, and 4$\mu \mathrm{m}$ sections were made of all spots for the 15 patients. After deparaffinization, endogenous peroxidase activity was blocked by $0.3 \% \mathrm{H}_{2} \mathrm{O}_{2}$ incubation for $30 \mathrm{~min}$. Macrophages were identified using monoclonal mouse antihuman antibody CD68 (1:50; clone PG-M1 [Dako]) for $60 \mathrm{~min}$ at room temperature. Endothelial cells were made visible using mouse antihuman anti-CD31 monoclonal antibody (1:25) clone JC70A (Dako) for $60 \mathrm{~min}$ at room temperature. To visualize areas of decreased oxygen level-that is, HIF- $1 \alpha$ - stainings were performed with mouse antibody HIF- $1 \alpha$ 67sup (Abcam) overnight at $4^{\circ} \mathrm{C}(20)$. Labeled polymer-horseradish peroxidase antimouse from the Dako K4006 kit was used as second antibody. Color reaction was developed using 3,3'-diaminobenzidine staining with chromogen, and sections were counterstained with hematoxylin. Nuclei were therefore stained blue, and specific immunostaining was shown as brown. Immunoreactivity in the plaques was scored by 2 independent observers as follows: 0 , none; 1 , mild; 2 , moderate; and 3 , extensive (21).

\section{Immunofluorescence}

To demonstrate colocalization of FR- $\beta$ with macrophages, fluorescence microscopy with CD68 and FR- $\beta$ was performed on hot spots and cold spots of atherosclerotic carotid plaque, determined with the IVIS Spectrum. After deparaffinization, antigen retrieval with $10 \mathrm{mM}$ tris-HCL plus $1 \mathrm{mM}$ ethylenediaminetetraacetic acid was performed for $60 \mathrm{~min}$. Subsequently, endogenous peroxidase activity was blocked by $0.3 \% \mathrm{H}_{2} \mathrm{O}_{2}$ incubation for $30 \mathrm{~min}$. Macrophages were identified using the same antibody, concentration, and incubation time as used with immunohistochemistry. FR- $\beta$ was visualized using biotinylated antihuman FR- $\beta$ antibody (1:10) overnight at $4^{\circ} \mathrm{C}$ (R\&D Systems). Tetramethyl rhodamine isothiocyanate (TRITC)-goat antimouse IgG3 (Southern Biotech) and streptavidine-APC were used as secondary antibodies. 4',6-diamidino-2-phenylindole (DAPI) staining was performed to stain nuclei. Pictures were taken using the Quantimet equipment (600S; Leica).

\section{RNA Isolation, Complementary DNA Synthesis, and Quantitative RT-PCR}

In this study, it appeared that RNA isolation from the imaged plaques was not possible because of the breakdown of RNA during folate-FITC incubation at room temperature. During the process of incubation for $30 \mathrm{~min}$, RNA remained stable (and detectable) with RT-PCR for only a few minutes. To investigate messenger RNA expression of relevant proteins in atherosclerotic plaques, we used biopsy samples from atherosclerotic plaques from the carotid artery directly stored at $-80^{\circ} \mathrm{C}$ immediately after explantation; these samples had not been imaged in the IVIS Spectrum. RNA isolation, complementary DNA synthesis, and quantitative RT-PCR were performed. In short, TRIzol (Invitrogen) was added to the frozen tissues, which were then ground with a pestle. Total RNA concentrations were determined using a ND-1000 spectrophotometer (NanoDrop Technologies). The RT step was performed using reagents from Invitrogen. One microgram of total RNA was transcribed to complementary DNA with $0.5 \mu \mathrm{L}$ of $7.5 \mu \mathrm{M}$ Oligo (dT) 24 primers, $1 \mu \mathrm{L}$ of M-MLV RT enzyme, $5 \times$ first-strand buffer, $0.9 \mu \mathrm{L}$ of $0.1 \mathrm{mM}$ dithiothreitol, and $25 \mathrm{mM}$ deoxyribonucleotide triphospate in a total volume of $25 \mu \mathrm{L}$ of RNase-free water. For the measurement of messenger RNA, $1 \mu \mathrm{L}$ of complementary DNA sample was added in duplicate for amplification by the Taqman RT-PCR system (ABI Prism 7900HT Sequence Detection System; Applied Biosystems). The investigated genes were glyceraldehyde-3-phosphate dehydrogenase (GAPDH), CD68, CD31, HIF$1 \alpha, \mathrm{HIF}-2 \alpha$, vascular endothelial growth factor (VEGF), folate receptor 1 or $\alpha$ (FR- $\alpha$ ), FR- $\beta$ (or folate receptor 2 ), IL-8, matrix metalloproteinase 9 (MMP-9), and angiopoietin 1 and 2 (Ang-1 and Ang-2, respectively). Threshold cycle $(\mathrm{Ct})$ values were determined with the software program SDS 2.3 (Applied Biosystems). Samples that needed more than 30 cycles of GAPDH (reference gene) before reaching the threshold were discarded. Relative gene
FIGURE 1. Ex vivo fluorescence imaging of human atherosclerotic plaques. (A) Fluorescence signals reflecting folate-FITC uptake occurring in specific areas of plaque intraluminally (upper) and extraluminally (bottom): incubation in phosphate-buffered saline (left), autofluorescence (middle), incubation with folate-FITC (right). (B) Intensity of fluorescence signals of plaques incubated with folate-FITC (solid bars) were increased both intraluminally and extraluminally when compared with autofluorescence signals (open bars). ${ }^{*} P<0.001$. ${ }^{\star \star} P<0.001$. FR $=$ folate receptor.
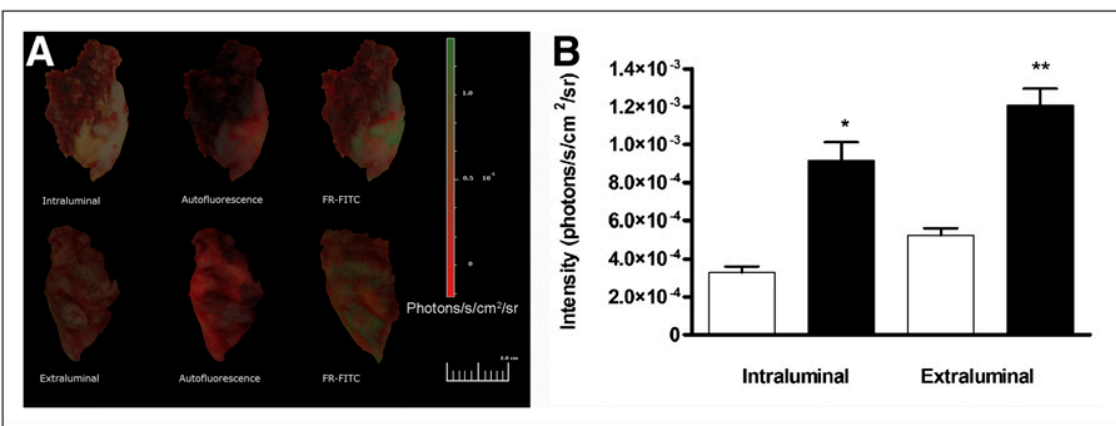
expression was normalized to the expression of GAPDH and calculated by the following formula: relative gene expression $=2^{-\Delta \mathrm{Ct}}$ $(\Delta \mathrm{Ct}=\mathrm{Ct}$ gene of interest $-\mathrm{Ct}$ GAPDH). Furthermore, samples of nonatherosclerotic umbilical artery were used as a control.

\section{Statistics}

Values are presented as mean $\pm \mathrm{SD}$. For comparison of fluorescence efficiency measurements in the IVIS Spectrum, a 2tailed, paired Student $t$ test was used for parametric distributions. Nonpaired continuous variables with a nonparametric distribution were analyzed using the Mann-Whitney $U$ test. For correlations, Pearson and Spearman correlation coefficients were used when appropriate. A 2 -sided $P$ value of less than 0.05 was considered statistically significant. Statistical tests were done with the Statistical Package for the Social Sciences (version 16.0; SPSS Inc.).

\section{RESULTS}

\section{Patient Characteristics}

Seventeen men and 3 women, with a mean age of $68.7 \pm$ $9.4 \mathrm{y}$, were included. Twenty carotid plaques were obtained during surgery. Patient characteristics are shown in Table 1.

\section{Fluorescence Imaging and Validation of Folate- FITC Labeling}

During fluorescence imaging of the plaques using the IVIS Spectrum, the fluorescence signals reflecting the folate-FITC uptake occurred in specific areas of the plaques (Fig. 1A). Fluorescence signals increased after $30 \mathrm{~min}$ of incubation with folate-FITC and reached a plateau phase after 30 min (Supplemental Fig. 1). When this plateau was reached, the final images for comparison were taken. There was a heterogeneous distribution of fluorescence signals across the plaque's surface, both at the intraluminal and at the extraluminal surfaces. The mean background fluorescence (radiance, photons $/ \mathrm{s} / \mathrm{cm}^{2} / \mathrm{sr}$ ) as measured before incubation with folate-FITC was $1.5 \mathrm{e}^{-3} \pm 7.4 \mathrm{e}^{-4}$ intraluminally and $2.5 \mathrm{e}^{-3} \pm 9.7 \mathrm{e}^{-4}$ extraluminally $(P<$ 0.001). After incubation with folate-FITC, these values were $9.8 \mathrm{e}^{-3} \pm 2.6 \mathrm{e}^{-2}$ (intraluminally) and $5.5 \mathrm{e}^{-3} \pm 0.4 \mathrm{e}^{-3}$ (extraluminally) $(P<0.001)$. Folate-FITC and background fluorescence ratios did not differ significantly between the 2 sides of the plaque. There was a significant difference in intensity between background signals and folate-FITC intraluminally and extraluminally $(P<0.001$, Fig. 1B).

To validate the folate-FITC signal, CD11b cells were stained for FR- $\beta$. In isolated mononuclear cells from the synovial fluid of a rheumatoid arthritis patient, a large population of macrophages was found (63\% were CD11b-positive cells). Staining CD11b-positive cells for FR- $\beta$ shows that most of the activated macrophages express FR- $\beta$ (Fig. 2). Staining with folate-FITC gives a high percentage of positive cells, which can be blocked with free folic acid, indicating that this is a specific interaction between FR- $\beta$ and folate-FITC.

\section{Immunohistochemistry and Immunofluorescence}

Hot, medium, and cold spots of 15 patients were stained with CD68 to identify macrophages in conjunction with folate-FITC uptake. Spots were identified as follows: cold spots exhibited low to no uptake, medium spots showed moderate uptake, and hot spots displayed high uptake of folate-FITC. Figure 3 shows a representative example of cold and hot spots with respect to CD68 staining, along with quantification of the staining intensities at the cold, medium, and hot spots. In Figure 4, staining for HIF-1 $\alpha$ in these identical spots is depicted. HIF-1 $\alpha$ upregulation was significantly increased in the hot spot, compared with the cold spot $(P=0.005)$. As a control, expression of CD31positive cells for the presence of endothelial cells was found heterogeneously on all plaque specimens, and there was no difference between hot, medium, or cold spots con-
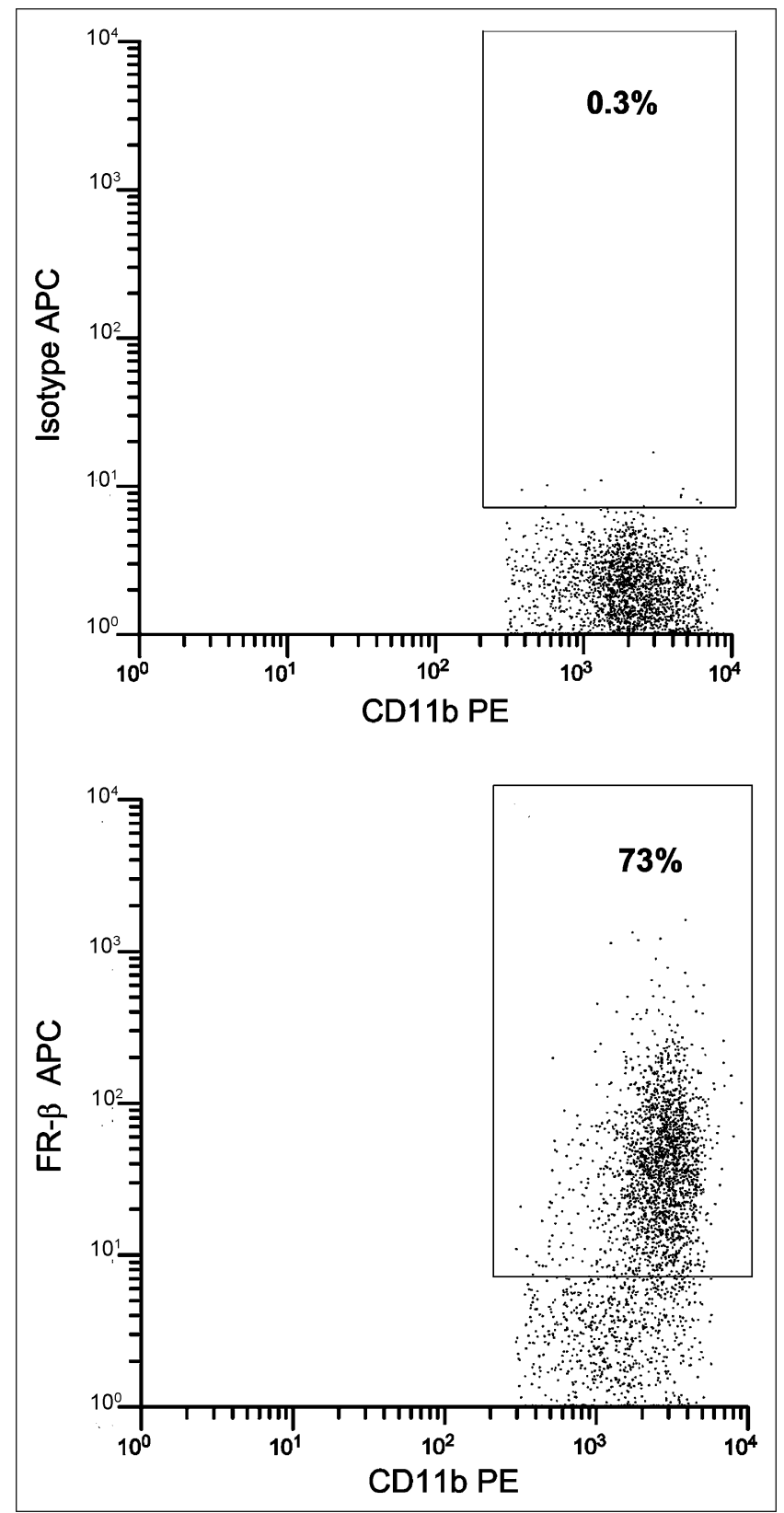

FIGURE 2. Flow cytometric analysis of macrophages from synovial fluid of a rheumatoid arthritis patient. Of this macrophage population, $73 \%$ express FR- $\beta$. 


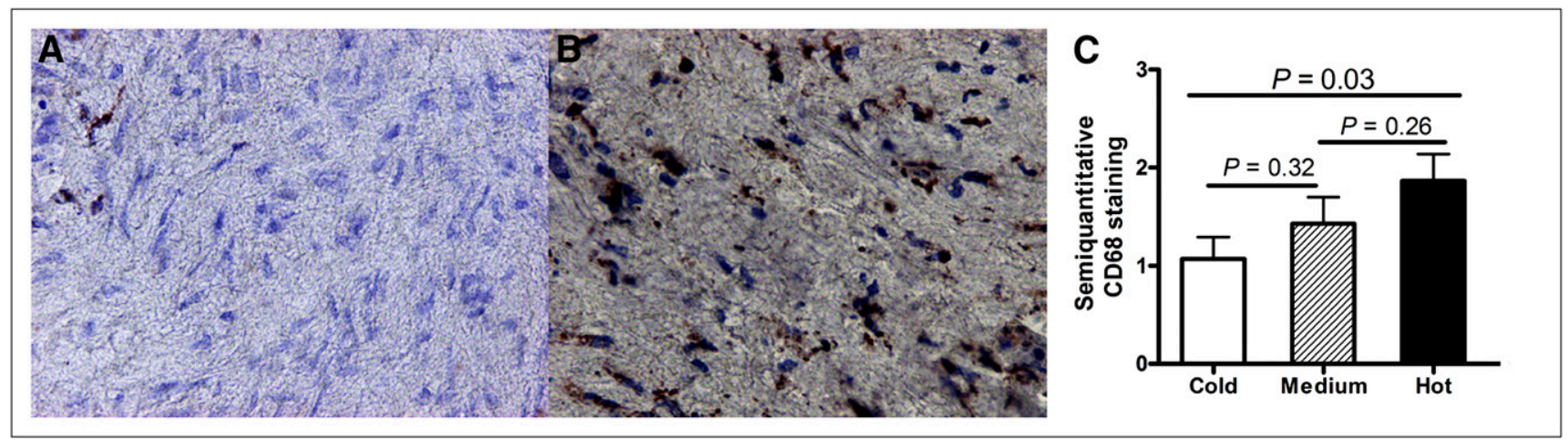

FIGURE 3. CD68 expression in atherosclerotic plaques of carotid arteries. (A) CD68-positive macrophages were nearly absent in cold spot of plaque. (B) Extensive CD68-positive macrophage expression is present in hot spot of plaque. (C) Immunoreactivity score of CD68 was increased in hot spots (solid bar) vs. cold spots (open bar) in 15 patients $(P=0.03$ ).

cerning expression of endothelial cells $(P=0.89)$. To demonstrate colocalization of macrophages and FR- $\beta$ in hot spots, double staining using immunofluorescence with CD68 and FR- $\beta$ was performed. Indeed, substantial colocalization of CD68 and FR- $\beta$ expression was encountered, demonstrating the presence of FR- $\beta$ on most macrophages in hot spots with DAPI staining of nuclei, TRITC staining of CD68 expression, a macrophage marker in red, and APC staining for FR- $\beta$ expression in green (Fig. 5).

\section{Messenger RNA Expression}

In 15 snap-frozen biopsy samples of atherosclerotic plaques and 3 control artery samples, messenger RNA levels were determined for FR- $\alpha$ and $-\beta$, CD68, and CD31 (Fig. 6A) and HIF-1 $\alpha$, HIF-2 $\alpha$, VEGF, MMP-9, Ang-1, Ang-2, and IL-8 (Fig. 6B). As expected, expression of the tumor-specific FR- $\alpha$ was not detected in the biopsy samples. Compared with control tissue, messenger RNA expression of FR- $\beta$, CD68, and MMP-9 was significantly increased, compared with control tissue $(P=0.02,0.01$, and 0.01 , respectively). FR- $\beta$ expression, moreover, significantly correlated with expression of the activated macrophage marker CD68 ( $r=0.53, P=0.045)$ (Fig. 7). Also, CD68 expression showed a positive correlation with HIF-1 $\alpha$ expression $(r=0.55, P=0.034)$ and MMP-9 expression $(r=0.63, P=0.021)$ (Supplemental Fig. 3). CD31 expression was positively correlated with VEGF $(r=$ $0.66, P=0.007)$ and Ang-1 expression $(r=0.63, P=$ $0.039)$. No clear correlation could be found between FR- $\beta$ and HIF1- $\alpha(r=0.07, P=0.13)$ or between VEGF and Ang-1 $(r=0.15, P=0.26)$.

\section{DISCUSSION}

In this study, we demonstrated the high expression of FR$\beta$ in the plaques of patients with severe atherosclerotic disease using a novel methodology for mapping biomarker activity with optical folate-FITC imaging and confirmed our results histologically and by RT-PCR. Folate-FITC specifically binds to folate receptors and in this context to the FR- $\beta$, which is a marker for inflammation as is specifically expressed on activated macrophages $(10,12,22)$. Moreover, the FR- $\beta$ on the cell surface of activated macrophages can bind folate-conjugated fluorophores with high affinity $(12,22)$. The significant difference between background fluorescence and folate-FITC uptake at both the intraand the extraluminal sides of the plaque might confirm the actual folate-FITC uptake in the plaque by ex vivo incubation, which was found to be heterogeneously distributed. Furthermore, a significant difference between the number

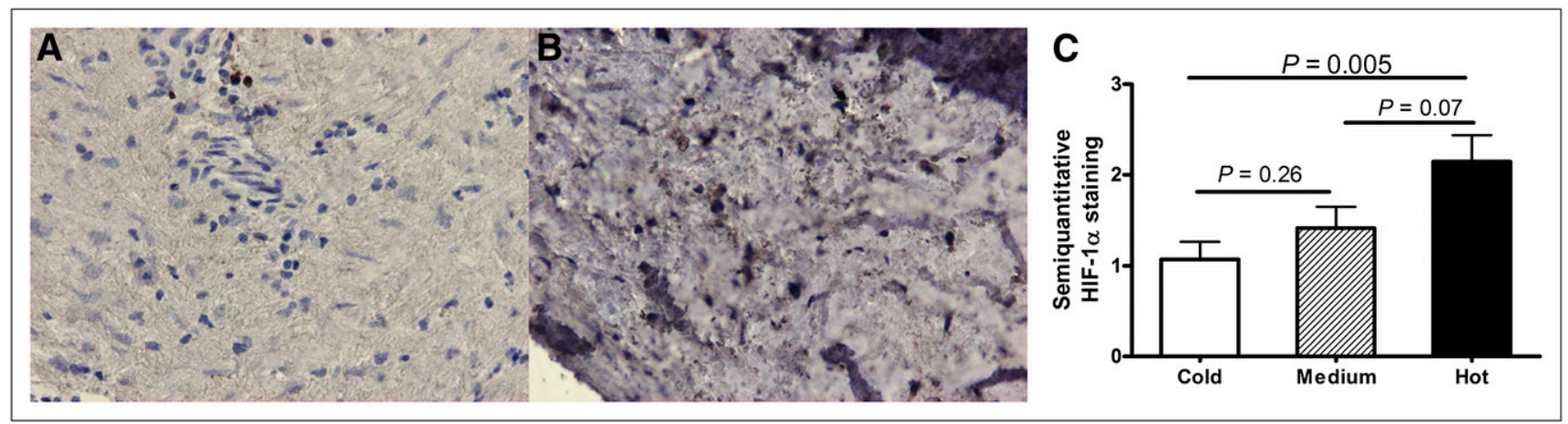

FIGURE 4. HIF-1 $\alpha$ expression in atherosclerotic plaques of carotid arteries. (A) Cold spot of plaque showed mild or no hypoxia. (B) Hot spot of plaque showed extensive hypoxia. (C) Immunoreactivity score of hypoxia was increased in hot spots (solid bar) vs. cold spots (open bar) measured in 15 patients $(P=0.005)$. 


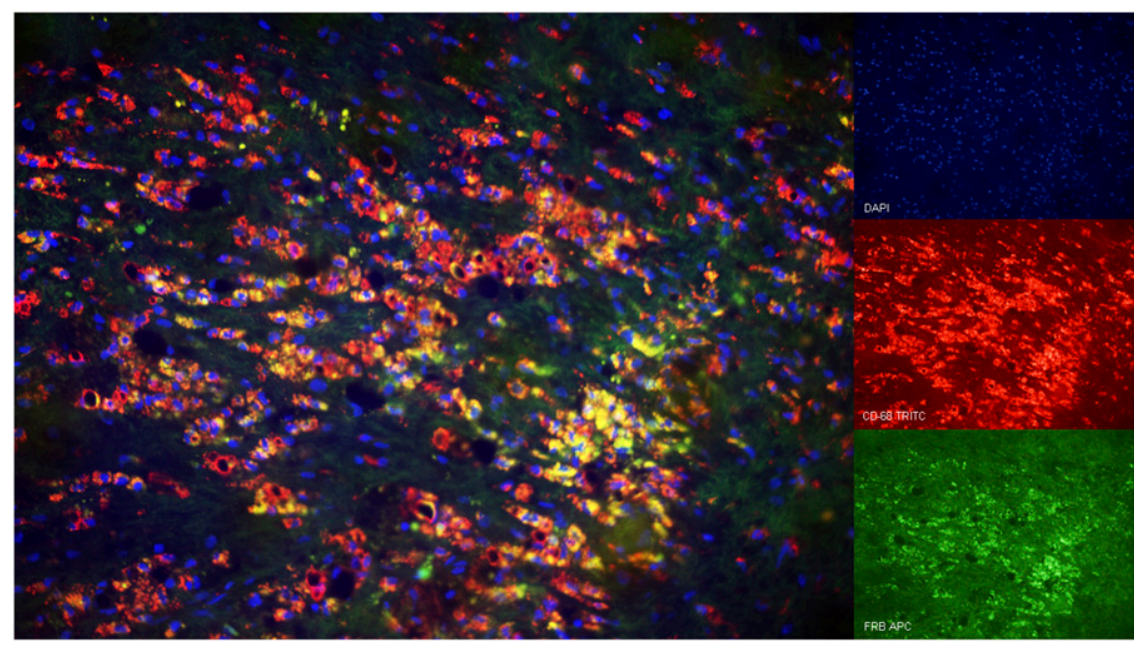

FIGURE 5. Colocalization of FR- $\beta$ and macrophages in hot spot of plaque from carotid artery. Blue = DAPI staining of nuclei; red $($ TRITC) = CD68 expression, macrophage marker; green (APC) $=$ FR- $\beta$ expression. Overlay of yellow represents colocalization of CD68 and FR- $\beta$. of activated macrophages in cold, medium, and hot spots demonstrated by mapping of folate-FITC uptake could be detected, indicating increased inflammation in hot spots. In a previous study, an excess of macrophages over vascular smooth muscle cells was found in plaques vulnerable to rupture (23). In a study by Sluimer et al., the presence of macrophages correlated with hypoxia and levels of HIF- $1 \alpha$ (21). Evidence of hypoxia in vascular disease is supported by in vivo detection of hypoxia in macrophage-rich regions in rabbit atherosclerosis ( 6 ). These reports are supported by our findings on the HIF- $1 \alpha$ stainings, which exhibited significant differential expression in cold, medium, and hot spots and correlated with the number of activated macrophages. This new approach of macrophage detection by folate-FITC labeling seems feasible to detect activated macrophages by their increased expression of FR- $\beta$ and is supported by double staining experiments (Fig. 5). In addition to morphologic and functional (duplex ultrasound) imaging, imaging of vulnerable plaques by means of activated macrophages might contribute to a better risk assessment and selection of patients at risk for ischemic stroke.
Activated macrophages have FR- $\beta$ on their surface and produce MMP-9. Symptomatic plaques showed a high relative gene expression of MMP-9 (Fig. 6B) and correlated with CD68 expression. This finding is in accordance with previous research, in which overexpression of MMP-9 in plaques had a strong association with unstable plaques and with the presence of macrophages within these plaques $(17,24)$. Furthermore, analysis of the presence of MMP-9 protein by enzyme-linked immunosorbent assay within excised carotid plaques revealed a high MMP-9 protein mass in calcified segments at or near the carotid bifurcation and in segments with intraplaque hemorrhage (18). Because MMP-9 also can be visualized using the IVIS technique as shown earlier by our group, this analysis could be combined with dual visualization of MMP-9 and FR- $\beta$, leading to a better assessment of inflammatory sites in vulnerable atherosclerotic plaques (18).

Endothelial cells produce VEGF and angiopoietins. The Ang-2 expression in the plaques, compared with Ang-1, was relatively high. This finding is in agreement with previous findings in which Ang-1 induced formation of stable vessels,

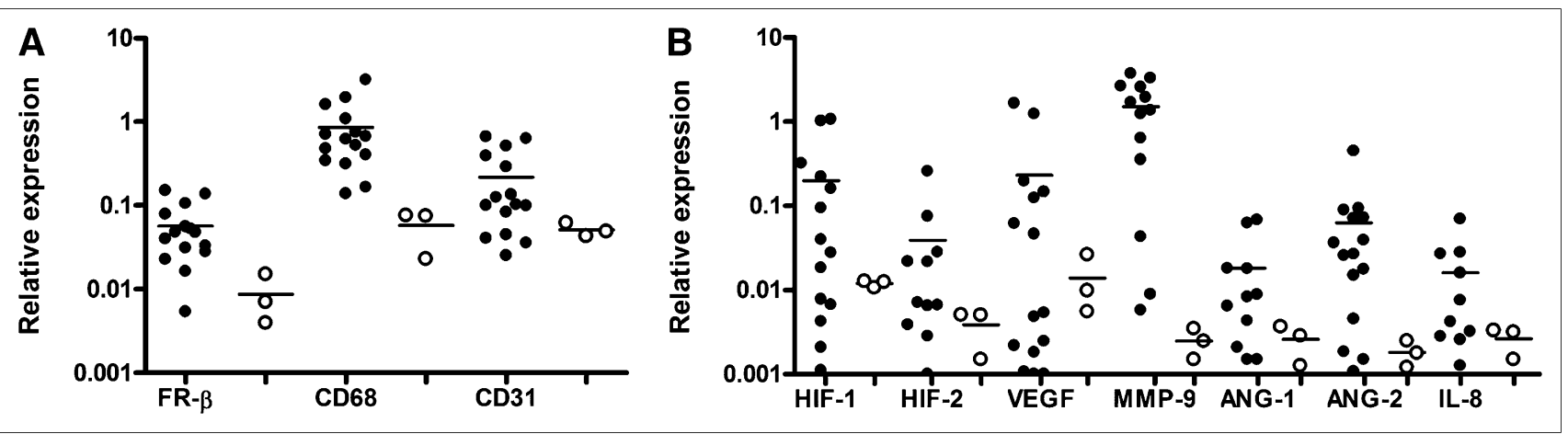

FIGURE 6. Relative expression of messenger RNA levels in 15 atherosclerotic plaques and 3 control artery samples. Quantitative RT-PCR was used to determine relative expression of different genes in human atherosclerotic plaques $(\bullet)$, compared with control artery tissue $(\bigcirc)$. (A) Messenger RNA expression of FR- $\beta$ and CD68 was significantly increased in atherosclerotic tissue, compared with control tissue $(P=$ 0.02 and 0.01 , respectively). (B) Messenger RNA expression of all inflammatory markers was higher in atherosclerotic tissue than in control tissue. 


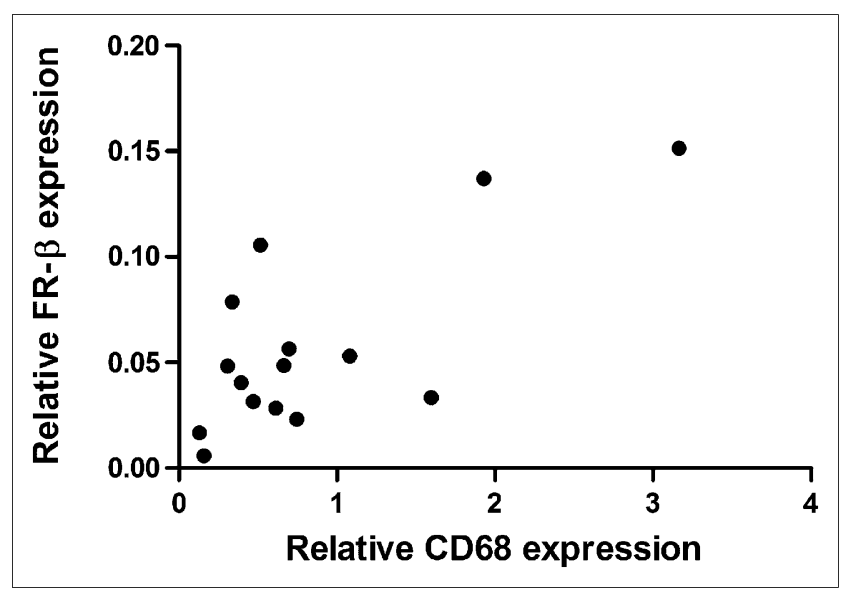

FIGURE 7. Correlation between messenger RNA levels of different genes in 15 human atherosclerotic plaques and 3 control artery samples. CD68 expression vs. FR- $\beta$ expression was determined by RT-PCR. Significant correlation was observed between CD68 expression and FR- $\beta$ expression $(P=0.045)$.

whereas Ang-2 destabilized the interaction between endothelial cells and their supporting cells (25). In the current study, CD31 correlated positively with VEGF and angiopoietin expression. As was expected, CD31 did not correspond with the FR- $\beta$ imaging because endothelial cells do not express folate receptors (Low, personal observations, 2010).

Fluorescence imaging using a folate-FITC optical contrast agent probe can visualize the distribution of activated macrophages and might therefore be a good marker of inflammation and vulnerability of human atherosclerotic plaques. However, there are several limitations to this study. The number of carotid plaques was relatively small. Moreover, the IVIS Spectrum equipment used in the current ex vivo setup cannot be applied for in vivo use in humans. FITC was used because it is currently approved for intravenous use in targeting the FR- $\alpha$ as a potential useful probe in oncology patients. Current applications for noninvasive optical imaging of fluorescent signals within a human carotid artery are limited by the use of fluorophores with emission wavelengths within the range of 450-600 nm such as FITC, leading to a limited penetration depth. Therefore, sophisticated imaging devices such as multispectral optoacoustic tomography for imaging of near-infrared fluorophores (e.g., IRDye-CW800) conjugated to folate might solve this problem for future clinical applications. It is anticipated that such a device could also be used for clinical purposes for the identification of high-risk vulnerable atherosclerotic plaques (26). We recently started with folate- ${ }^{99 \mathrm{~m}} \mathrm{Tc}$ (kindly provided by Phillip S. Low) imaging. The results look promising; therefore, folate- ${ }^{99 \mathrm{~m}} \mathrm{Tc}$ might be a good compound for in vivo use in the future using the SPECT modality.

Furthermore, fluorescence imaging with the IVIS Spectrum as it is used in the current setup by definition is 2dimensional - that is, a hot spot on the extraluminal side is not per se a hot spot on the intraluminal side. Future ex vivo 3-dimensional rendering and more sensitive quantification of picomolar concentrations using fluorescence molecular tomography, as described by Ntziachristos et al., might solve this problem and is currently under investigation (27).

\section{CONCLUSION}

High FR- $\beta$ expression, as visualized by optical fluorescence imaging using folate-FITC, correlated with increased HIF- $1 \alpha$ staining, and a trend could be seen between high FR- $\beta$ expression and CD68, representing activated macrophages. In addition, FR- $\beta$ messenger RNA levels correlated with CD68, HIF- $1 \alpha$, and MMP-9 messenger RNA expression. Molecular imaging of the pathogenesis related to the vulnerability of atherosclerotic plaques might enable the clinician to make more substantiated therapeutic decisions in the treatment of patients with carotid artery disease in the future.

\section{DISCLOSURE STATEMENT}

The costs of publication of this article were defrayed in part by the payment of page charges. Therefore, and solely to indicate this fact, this article is hereby marked "advertisement" in accordance with 18 USC section 1734.

\section{ACKNOWLEDGMENT}

No potential conflict of interest relevant to this article was reported.

\section{REFERENCES}

1. Lindholm LH, Mendis S. Prevention of cardiovascular disease in developing countries. Lancet. 2007;370:720-722.

2. Antohe F. Endothelial cells and macrophages, partners in atherosclerotic plaque progression. Arch Physiol Biochem. 2006;112:245-253.

3. Ringleb PA, Chatellier G, Hacke W, et al. Safety of endovascular treatment of carotid artery stenosis compared with surgical treatment: a meta-analysis. $J$ Vasc Surg. 2008;47:350-355.

4. Zeebregts CJ, Meerwaldt R, Geelkerken RH. Carotid artery stenting: a 2009 update. Curr Opin Cardiol. 2009;24:528-531.

5. Wallis de Vries BM, van Dam GM, Tio RA, Hillebrands JL, Slart RH, Zeebregts CJ. Current imaging modalities to visualize vulnerability within the atherosclerotic carotid plaque. J Vasc Surg. 2008;48:1620-1629.

6. Nighoghossian N, Derex L, Douek P. The vulnerable carotid artery plaque: current imaging methods and new perspectives. Stroke. 2005;36: 2764-2772.

7. Fuster V, Lewis A. Conner Memorial Lecture. Mechanisms leading to myocardial infarction: insights from studies of vascular biology. Circulation. 1994;90:2126-2146

8. Tiwari RL, Singh V, Barthwal MK. Macrophages: an elusive yet emerging therapeutic target of atherosclerosis. Med Res Rev. 2008;28:483-544.

9. Choudhury RP, Lee JM, Greaves DR. Mechanisms of disease: macrophagederived foam cells emerging as therapeutic targets in atherosclerosis. Nat Clin Pract Cardiovasc Med. 2005;2:309-315.

10. Xia W, Hilgenbrink AR, Matteson EL, Lockwood MB, Cheng JX, Low PS. A functional folate receptor is induced during macrophage activation and can be used to target drugs to activated macrophages. Blood. 2009;113:438-446.

11. Ayala-López W, Xia W, Varghese B, Low PS. Imaging of atherosclerosis in apoliprotein e knockout mice: targeting of a folate-conjugated radiopharmaceutical to activated macrophages. J Nucl Med. 2010;51:768-774.

12. Paulos CM, Turk MJ, Breur GJ, Low PS. Folate receptor-mediated targeting of therapeutic and imaging agents to activated macrophages in rheumatoid arthritis. Adv Drug Deliv Rev. 2004;56:1205-1217.

13. van Dam GM, Themelis G, Crane LM, et al. Intraoperative tumor-specific fluorescence imaging in ovarian cancer by folate receptor-alpha targeting: first inhuman results. Nat Med. 2011;17:1315-1319. 
14. Luque A, Turu M, Juan-Babot O, et al. Overexpression of hypoxia/inflammatory markers in atherosclerotic carotid plaques. Front Biosci. 2008;13:6483-6490.

15. Vink A, Schoneveld AH, Lamers D, et al. HIF-1 alpha expression is associated with an atheromatous inflammatory plaque phenotype and upregulated in activated macrophages. Atherosclerosis. 2007;195:e69-e75.

16. Westra J, Molema G, Kallenberg CG. Hypoxia-inducible factor-1 as regulator of angiogenesis in rheumatoid arthritis: therapeutic implications. Curr Med Chem. 2010;17:254-263.

17. Brevetti G, Silvestro A, Schiano V, Chiariello M. Endothelial dysfunction and cardiovascular risk prediction in peripheral arterial disease: additive value of flow-mediated dilation to ankle-brachial pressure index. Circulation. 2003;108: 2093-2098.

18. Wallis de Vries BM, Hillebrands JL, van Dam GM, et al. Images in cardiovascular medicine: multispectral near-infrared fluorescence molecular imaging of matrix metalloproteinases in a human carotid plaque using a matrix-degrading metalloproteinase-sensitive activatable fluorescent probe. Circulation. 2009;119: e534-e536.

19. Kinne RW, Brauer R, Stuhlmuller B, Palombo-Kinne E, Burmester GR. Macrophages in rheumatoid arthritis. Arthritis Res. 2000;2:189-202.

20. Brouwer E, Gouw AS, Posthumus MD, et al. Hypoxia inducible factor-1-alpha (HIF-1alpha) is related to both angiogenesis and inflammation in rheumatoid arthritis. Clin Exp Rheumatol. 2009;27:945-951.
21. Sluimer JC, Gasc JM, van Wanroij JL, et al. Hypoxia, hypoxia-inducible transcription factor, and macrophages in human atherosclerotic plaques are correlated with intraplaque angiogenesis. J Am Coll Cardiol. 2008;51:12581265 .

22. Müller C, Forrer F, Schibli R, Krenning EP, de Jong M. SPECT study of folate receptor-positive malignant and normal tissues in mice using a novel ${ }^{99 \mathrm{~m}} \mathrm{Tc}$ radiofolate. J Nucl Med. 2008;49:310-317.

23. Newby AC, George SJ, Ismail Y, Johnson JL, Sala-Newby GB, Thomas AC. Vulnerable atherosclerotic plaque metalloproteinases and foam cell phenotypes. Thromb Haemost. 2009;101:1006-1011.

24. Alvarez B, Ruiz C, Chacon P, Alvarez-Sabin J, Matas M. Serum values of metalloproteinase- 2 and metalloproteinase- 9 as related to unstable plaque and inflammatory cells in patients with greater than $70 \%$ carotid artery stenosis. $J$ Vasc Surg. 2004;40:469-475.

25. Post S, Peeters W, Busser E, et al. Balance between angiopoietin-1 and angiopoietin-2 is in favor of angiopoietin-2 in atherosclerotic plaques with high microvessel density. $J$ Vasc Res. 2008;45:244-250.

26. Razansky D, Harlaar NJ, Hillebrands JL, et al. Multispectral optoacoustic tomography of matrix metalloproteinase activity in vulnerable human carotid plaques. Mol Imaging Biol. 2012;14:277-285.

27. Ntziachristos V, Razansky D. Molecular imaging by means of multispectral optoacoustic tomography (MSOT). Chem Rev. 2010;110:2783-2794. 